\title{
What other sciences look like ${ }^{1}$
}

\author{
Josep M. Colomer
}

University Pompeu Fabra, Barcelona

\begin{abstract}
In order to have references for discussing mathematical menus in political science, I review the most common types of mathematical formulae used in physics and chemistry, as well as some mathematical advances in economics. Several issues appear relevant: variables should be well defined and measurable; the relationships between variables may be non-linear; the direction of causality should be clearly identified and not assumed on a priori grounds. On these bases, theoretically-driven equations on political matters can be validated by empirical tests and can predict observable phenomena.
\end{abstract}

Keywords: natural and social sciences, econometrics, political science methods, mathematical models, regression analysis. 


\section{INTRODUCTION}

That the sciences of nature -- physics, chemistry, astronomy -- should be the model for the sciences of society is an old postulate. Economists, in particular, have adopted this perspective at least since William S. Jevons proposed to construct a 'social physics' in the late nineteenth century. Many political scientists, in turn, have looked to economics as the model for their own endeavours, in great part precisely because economics has developed such kind of knowledge. Nowadays, almost nobody believes that 'natural' laws exist in society. But the outcomes of human interactions can produce regularities amenable to being captured by mathematical formulae similar to those used by physicists or by economists. Political scientists may expect a set of relevant postulates, if they are captured by a few stylised formulae, to be the foundations of a deductive method of inquiry. Well-modelled postulates, if expressed as mathematical relationships between well-defined variables, can be subjected to empirical testing, and are capable of being used to develop specific predictions.

In the following, I first review the kind of equations typically used in physics and chemistry. Then, some traditional objections to extending the natural science model to economics are discussed. A set of basic characteristics of mathematical equations to be shared in political science is enunciated. Finally, two examples are reviewed, respectively focusing on a deductive analysis of economic laws and on some drawbacks of certain inductive empirical exercises in political science.

\section{PHYSICS AND CHEMISTRY EQUATIONS}

The mathematical menus used in the natural sciences, which are in contrast to the most common ones in economics and political science, can be briefly surveyed. Let us start with a number of standard mathematical formulae used in physics. In a recent survey, the readers of a scientific monthly magazine were asked to select 'the greatest equations ever'. Let us remark that an equation proper is a formula that establishes relationships among variables; once tested, it is considered that it states observed facts and is thus empirically true. The respondents considered simplicity, practicality and historical relevance as criteria of choice - potentially a good set of criteria also for political scientists. From 120 responses, the twenty most popular equations in physics are those in Table 1, with Maxwell's equations of electromagnetism and the Euler equation at the top (Crease, 2004; see also Farmelo, 2002).

This list of physics equations can be taken as a benchmark to identify crucial properties of mathematical formulae that should also be found in other disciplines with similar scientific ambition. Most equations include only a few variables (typically around three), and even fewer constants. All the variables involved -- time, space, matter, temperature, pressure etc -- are well defined and can be measured, usually with well-established instruments. Very few formulae are simply sums. Specifically, among these top twenty equations, fourteen use multiplication, four use division, five use powers, and six use derivatives, while only five use addition or subtraction (and only once as the only operation, in the beautiful but trivial $1+1=2$ ). Nine of twenty equations follow the same pattern: any variable can be obtained by multiplication/division of one or more other variables times possibly a constant. The most common format is $y=a \Pi x_{i}^{b i}$, where the exponents $b_{i}$ are simple integers or fractions, positive or negative, 
and $a$ is a constant. This relationship implies an additive linear format for the logarithms of the variables: $\log y=\log a+\sum b_{i} \log x_{i}$.

Table 1. Greatest Equations in Physics

Euler's equation

Maxwell's equations

Newton's Second Law

Pythagorean theorem

Schrödinger's equation

Einstein's equation

Boltzmann equation

One plus one

Principle of least action

DeBroglie's equation

Fourier transform

Einstein's general theory

of relativity

Circumference of a circle

Dirac equation

Riemann zeta function

Hubble's Law

Simplest ratio

Ideal gas law

Balmer series

Planck's equation

$$
\mathrm{e}^{\mathrm{i} \pi}=-1
$$$$
\nabla . \mathrm{D}=\rho, \nabla . \mathrm{B}=0, \nabla \times \mathrm{E}=\partial \mathrm{B} / \partial \mathrm{t}, \nabla \times \mathrm{H}=\partial \mathrm{D} / \partial \mathrm{t}+\mathrm{J}
$$

$$
\mathrm{F}=\mathrm{ma}
$$$$
\mathrm{a}^{2}+\mathrm{b}^{2}=\mathrm{c}^{2}
$$

$\mathrm{H} \Psi=\mathrm{E} \Psi$

$$
\mathrm{E}=\mathrm{mc}^{2}
$$$$
\mathrm{S}=\mathrm{k} \ln \mathrm{W}
$$

$1+1=2$

$\delta \mathrm{S}=0$

$\mathrm{p}=\mathrm{h} / \lambda$

$f(x)=\int f(k) e^{2 \pi k x} d k$

$\mathrm{G}_{\mu \nu}=8 \pi \mathrm{GT}_{\mu \nu}$

$\mathrm{C}=2 \pi \mathrm{r}$

$\mathrm{i} \gamma . \partial \Psi=\mathrm{m} \Psi$

$\zeta(\mathrm{s})=\Pi\left[\mathrm{p}^{\mathrm{s}} /\left(\mathrm{p}^{\mathrm{s}}-1\right)\right]$

$\mathrm{v}=\mathrm{H}_{0} \mathrm{~d}$

$\mathrm{a} / \mathrm{b}=\mathrm{c} / \mathrm{d}$

$\mathrm{PV}=\mathrm{nRT}$

$1 / \lambda_{\mathrm{n}}=\mathrm{R}\left[1 / 2^{2}-1 / \mathrm{n}^{2}\right]$

$\mathrm{E}=\mathrm{h} v$

Source: Crease 2004. 
However, not all these properties of the greatest equations in physics are found in an apparently close discipline, chemistry. Equations in chemistry show the reactants and the products in a reaction. Several types of reaction are usually distinguished in chemistry textbooks (synthesis, decomposition, replacement, ionic), but all of them use only sum signs and arrows. An example is a reaction occurring when two ions come together to produce a precipitate, as in the formation of water:

$$
\mathrm{HCl}_{(\mathrm{aq})}+\mathrm{NaOH}_{(\mathrm{aq})} \rightarrow \mathrm{NaCl}_{(\mathrm{aq})}+\mathrm{H}_{2} \mathrm{O}_{(\mathrm{l})}
$$

- that is, acid and base form gas and water.

This type of equation represents a mere convention to symbolise reactions, and has no specific mathematical signification. Even the sign ' + ' could well be replaced with the expressions 'and' or ' $\&$ ' (instead of 'plus'), or even with 'times', ' $x$ ', since it only means that two or more reactants interact or two or more products are formed. The sign $\rightarrow$ means 'yields' and shows the direction of the action, while, in some equations, a double arrow $\leftrightarrow \rightarrow$ shows that the reaction is reversible and can go in both directions.

A number of properties can be noted. As in physics, the 'variables' included in chemical equations are well-identified 'elements' -- hydrogen, oxygen, magnesium, sodium, calcium etc. The equations show the quantities of each component, either reactants or products, which can be precisely measured with the usual instruments. Every chemical compound, formed by one or more 'elements', has a formula that cannot be altered. Every equation makes a precise prediction, which can be effectively tested.

However, in contrast to physics, mathematical signs in chemistry are relatively 'weak', since only ' + ' and arrows are used. No multiplications, divisions, powers or other mathematical operations are employed. On the other hand, chemical equations specify directionality well by using arrows (either in one or in two directions) instead of 'equal' '=' signs. In the above equation for the formation of water, for instance, the arrow is certainly not reversible: salt and water do not form acid and base (they produce only salty water). This suggests that the use of arrows might help to specify hypothetical lines of causality in other fields, including in political science models using standard regression techniques.

\section{'...Physics equations can be taken \\ as a benchmark to identify \\ crucial properties of mathematical \\ formulae that should also be found \\ in other disciplines with similar $s$ \\ cientific ambition.'}




\section{ECONOMETRICS}

The science of economics has developed, especially since the late nineteenth century, according to the model of the natural sciences. As in physics, and in contrast to still common uses in political science, economic theorems are not generalisations induced from experience. They postulate relationships among variables that should enable us to explain and predict economic observations. Economists began following this path several generations ahead of mainstream political scientists, but a few decades ago they still encountered difficulties and objections that were similar to those faced more recently within political science.

In particular, it has been argued that the depth and precision of scientific knowledge acquired in physics may not be achieved in economics for two reasons. One has to do with the range of validity of economic theorems. Economists and other social scientists typically suspect that the human world changes more than the natural one, thus imposing more constraining territorial and temporal limits on the validity of hypotheses and postulates. Kenneth Arrow remarked that while physical laws are 'true for all time', economics (and for that matter, political science) is more constrained by given circumstances. Accordingly, each historical or contemporary episode should 'be interpreted as the application of general principles to unique contexts' (Arrow, 1985).

Nevertheless, it can be observed that the laws of physics are also valid only under specified conditions. Galileo's law of falling bodies, for instance, implies an idealised 'perfect vacuum', but to measure and predict each specific episode, the resistance of air or 'friction' and other circumstances have to be estimated. Actually, physical laws do not predict the future in an unconditional sense. They merely say that if certain conditions are fulfilled, then certain outcomes can be expected. Whether this implies a difference of degree or of quality in the kind of knowledge that can be developed in the natural sciences and in the social sciences, is something open to discussion. To use Arrow's own comparison, it is likely that the social sciences should be able to develop, in proportion, more 'geology' than 'physics' or 'chemistry', that is, more study of specific events than standard laws. But no 'geology' -- that is, the study of business or public administration -- is feasible without solid foundations in 'physics' and 'chemistry' -- that is, in economics and political science.

A second objection is that in the social sciences there is a greater degree of influence of the observer on the subject being observed. Specifically, knowledge of economic phenomena may itself become an economic variable, since people with such knowledge can change the economic situation to which they refer. Again, influence of the observer on the observed has also been claimed for any science using laboratory experiments, since observation always means interaction. In quantum mechanics, for instance, 'seeing' particles means bombarding with photons, but it fails for subatomic particles.

It is clear, nevertheless, that this objection is more relevant to the development of testable postulates and predictions in the social sciences. By means of human action, a structural variable in a model can be manipulated to become an exogenous or 'independent' variable, with the intention of producing certain outcomes regarding other variables. An implication is that the line of hypothetical directionality should be specified in any model establishing relationships between structural or institutional variables. Another is that decisions likely to be made by human beings given specific 
constraints and incentives may be specified in order to account for expected outcomes -as developed in game theory, as well as in prospect theory and related approaches.

\section{TOWARDS A POLIMETRICS}

Rein Taagepera's invitation to develop new mathematical tools in political science can now be revisited. In the light of certain developments in economics alluded to above, a few lessons and a few precautionary warnings may emerge.

First, in general, economic theorems include, as in physics or chemistry, only a small number of variables and constants, which is not always the case in empirical studies in politics.

Second, coefficients can be measured. Econometrics has developed a set of tools to 'estimate' parameters in a model, starting from reasonable conjectures and progressing to increasingly broader empirical observations. It is said that frequent reestimates with new sets of data permit the 'calibration' of previous quantitative coefficients, without abandoning the aim of establishing adjusted values with broad validity. This is in sharp contrast with the scarce use of quantitative coefficients that are found in statistical analysis in politics when further analyses or predictions on the same subject are developed or related problems are explored.

Third, non-linear forms of equations are widely used in economics. I have found two early warnings regarding the narrowness of the traditional linear, additive regression model, respectively in economics and political science. They may illustrate the temporal gap in the development of more advanced statistical techniques in the two disciplines. Already in 1970, the economist E. Malinvaud was 'surprised to realize how little developed [was] the statistical theory of non-linear regression', while he invited exploration of the conditions under which non-linear regression performs well, and began doing it himself (Malinvaud, 1970).

About twenty-something years later, a comparable, although apparently unrelated claim was made in political science by James McGregor (1993). In order to show the drawbacks of the linear additive regression model, he took random data that fit perfectly three well-established laws in physics (Galileo's law of falling objects, Boyle's ideal gas law, and Newton's law of gravitational attraction) and analysed those data by regression. He concluded that 'none of the regression equations comes even close to capturing the real form of the underlying relationship'. McGregor rightly inferred that political scientists using such a technique may be blind to underlying relationships between political variables. Two types of error are possible as a consequence of focusing on regression analysis with the unquestioned assumption that the relationship to be revealed should be linear and additive. First, true relationships may be overlooked because, not being linear and additive, they cannot be revealed by the regression model (as in the case of physical laws just mentioned). Second, certain conclusions can be accepted as true (for example because a high $\mathrm{R}$ is found) when they are not, as one may suspect happens rather frequently in certain kinds of empirical political studies.

Finally, the direction of the action is relevant. This is not only because some relationships between structural variables may not make much sense in one of the directions, but because human decisions may alter any of the variables and make it 
exogenous or independent, as will be discussed below. The 'arrows' in a relationship should be specified.

\author{
'Actors' decisions regarding the \\ manipulation of some variables, \\ as well as the subsequent effect \\ of those decisions on structural \\ relationships, should occupy a central \\ place in social scientific analyses.'
}

\title{
A COMPARISON IN ECONOMICS AND POLITICAL SCIENCE
}

Let us discuss a couple of examples in economics and political science to show the role of the properties discussed above -- number of variables, measurement of parameters or coefficients, form of the equation, and directionality of the relationship.

An example of a mathematical equation in economics is Okun's law (from the economist Arthur Okun, 1962) which presents a relationship between changes in the rates of unemployment and output (usually measured as Gross Domestic Product). Quantitative estimates of the variables were initially identified on the basis of observing the United States' economy from 1965 or so, but broader observations in time and space have permitted more adjusted values. By regressing change in unemployment on output, it has been found that for every 3 percentage points increase in output about a 1 point change in the rate of unemployment can be expected (Prachowny, 1993).

Consider now another economic law, traditionally represented by the so-called Phillips curve (from economist A. W. Phillips, 1958), which postulates a trade-off between inflation and unemployment. Quantitative estimates of the variables were initially given on the basis of long-term data in the United Kingdom. Although so-called 'stagflation' in the 1970s challenged the relationship (because the two variables, inflation and unemployment, both increased), the Phillips curve has been re-vindicated since the 1990s in a US context of low levels and higher stability of both inflation and unemployment (roughly around 4 per cent).

There are two ways to read the Phillips curve. The 'classical' or 'monetarist' point of view implied that a decrease in unemployment would produce higher inflation, leading thus to the invitation to regress change in inflation on unemployment. A Keynesian reading, however, implied the opposite. If the government increases the quantity of money (through monetary of fiscal policy), while inflation may rise, it will reduce unemployment. The mathematical consequence is that different parameters can be obtained in the relationship between the two variables depending on which direction is used. The slope of the Phillips curve will be different if unemployment is regressed 
on inflation rather than inflation on unemployment. The Phillips curve appears to be flatter under the Keynesian direction (for instance, a decrease of 5 percentage points in unemployment may produce an increase of 5 points in inflation, but, under the same circumstances, only an increase of 10 points in inflation may be able to produce a 5 point decrease in unemployment; see Sargent, 1999).

That different coefficients and curve slopes are found depending on the direction of the relationship can be explained with the help of a theoretical model. Looking back at Okun's law referred to above, it seems clear that the 'Keynesian' direction implies a more complex and indirect relationship between the two variables, which can explain its lower effect. If the government increases the quantity of money, the immediate consequence is that it increases the amount that people spend. Higher spending increases the demand for goods and services, which leads firms to increase output. Higher production causes firms to contract more workers, as postulated by Okun's law. Thus, the increase in the quantity of money, while it can increase inflation, will reduce unemployment, at least in the short term -- not directly but through a succession of interactions that can make sense of the lower coefficient of the curve.

The relationship is not bidirectional, as in the implicit assumption:

$\nabla$ Unemployment $\leftarrow \rightarrow \Delta$ Inflation,

but rather unidirectional, as in the model suggested by Okun's law and the original Phillips' law:

\section{$\Delta$ Output $\rightarrow \nabla$ Unemployment $\rightarrow \Delta$ Inflation $\rightarrow \Delta$ Output $\rightarrow \ldots$}

This can be clarified with the appropriate mathematical tools. But if a bidirectional linear regression model is used, it is likely that analysts will be confused and policy-makers will be myopic. This is not very different from what happens in physics, where most phenomena correspond to two-directional equations, but there is 'hysteresis', where the system follows one path from one state to another but returns by a different path. The long-lasting controversy in the past between 'monetarists' and 'Keynesians' suggests that lack of appropriate statistical techniques probably hindered both sound understanding and efficient policy-making in this important field.

Let us now turn to an egregious example in political science. In order to explain and predict the results of the United States presidential elections several competing equations with different sets of independent variables have been proposed. A set of these equations, based on successive trials and errors, are regularly presented at the American Political Science Association annual meetings every election year (up to seven in 2004) and duly published and commented on in subsequent issues of the journal, $P S$.

The approach is totally inductive. Each of the equations presented by different authors typically selects a different set of 'independent' variables from which it tries to derive the share of the two-party popular vote for the candidates of the major parties. The most usual procedure is to test some selection of variables retrospectively for the US presidential elections for the period since the end of World War Two, apply the better-fitted coefficients to data for the current election year, make the corresponding prediction, revise the fit a posteriori, correct again the selection, the definition or the operationalisation of variables, find retrospectively the corresponding coefficients, and so on. This might correspond to and make good use of sound techniques introduced in econometrics to 'calibrate' initial parameters with additional data. This is indeed an effort to make progress from more traditional models that involved trying to forecast election results by regressing either solely on the state of the economy or solely on 
opinion polls, as well as regarding prediction markets or betting. (See an analysis of the relative performance of these different approaches in Leigh and Wolfers, 2006).

But it can be argued that these empirical exercises would work better if they were supported by some model or hypothesis about the likely relationship between relevant variables. In the set of exercises mentioned above, no initial model is discussed on the basis of reasonable assumptions or conjectures regarding voters' motivations and choices or regarding parties' or candidates' preferences or platforms. While there have been numerous changes in data, operationalisation and selection of variables, the only mathematical model that has been used so far is linear additive regression.

In the published exercises, almost all regression results are given in tabular form, not even as equations. One recent exception (Abramowitz, 2005) illustrates, however, the implicit model. Based on the results of all presidential elections since World War Two and a particular set of independent variables, Abramowitz obtains the following estimates:

$$
\mathrm{V}=50.3+0.81 * \mathrm{GDP}+0.113 * \mathrm{NETAPP}-4.7 * \mathrm{TFC},
$$

'where $\mathrm{V}$ is the predicted share of the major party vote for the incumbent party, GDP is the growth rate of real gross domestic product during the first two quarters of the year, NETAPP is the incumbent president's net approval rating in the final Gallup Poll in June, and TFC is the time-for-change dummy variable. TFC takes on the value of ' 0 ' if the president's party has controlled the White House for one term and ' 1 ' if the president's party has controlled the White House for two or more terms' (Abramowitz, 2005: 31).

In successive exercises, changes in the set and measurement of variables are driven by trial and error with statistical coefficients. As summarised by the editor of the latest issue:

Several of the models have been amended [since the previous election]. I [Campbell] adjusted the second quarter GDP growth for non-incumbent candidates of the in-party. Lockerbie simplified his number of terms variable and dropped the pre-election year income growth variable. Norpoth revised his primary performance variables and added a partisan baseline variable. Abramowitz changed his late June presidential approval measure. Wlezien and Erikson present a bracketed forecast using alternatively the approval ratings and now also the preference polls in their model. Holbrook used the mean presidential approval over the summer rather than spring months and weighted the Survey of Consumers retrospective personal finances measure by an index of economic news. Lewis-Beck and Tien dropped their 'peace and prosperity' index and added a job growth variable, an incumbent party advantage variable, and an interaction of incumbency and GNP growth (Campbell, 2004: 734-5).

So far, the models' predictive capabilities have remained modest. In 2000, one widely-read forecast gave Al Gore the victory, even stating that 'It's not even going to be close' (Washington Post, 26 May 2000, p.1; see Lewis-Beck and Tien, 2004: 753). In 2004 the average of seven forecasts was relatively closer to the actual result (although a fifty-fifty bet would not have been worse on this occasion). Perhaps in 2008 the seven or so different models will still compete with each other. Most of them will revise again their selection or operationalisation of variables and especially their coefficients. Or perhaps they may seek additional support from a previous, reasonable discussion about the criteria for choosing variables and the form of the equation. A reasoned model is 
still missing for why the impacts of relevant variables should be additive rather than multiply together or interact in still other ways.

\section{CONCLUSION}

There can still be a lot to learn in political science from the uses of mathematical models in other disciplines. Certain properties of the types of equations typically used in the natural sciences and in economics can be shared in political studies: First, a simple and relevant equation should include a small number of well-defined variables. Second, parameters and coefficients should be measured. Third, a mathematical equation should be based on reasonable hypotheses about the relationship among variables, which usually requires assumptions regarding actors' motives and choices. It may have a nonlinear form, but include multiplication, division, power or derivative (or have an additive linear format for the logarithms of the variables). Fourth, the directionality of the relationship should be specified (perhaps using 'arrows' instead of 'equal' signs).

Certain warnings already raised in economics should also be taken into account. Hypotheses and postulates regarding political outcomes are valid only in given circumstances, which can change for the observable set of phenomena under consideration (and more than is usual in the natural sciences). Thus, hypotheses should be postulated within explicit territorial and temporal limits. Actors' decisions regarding the manipulation of some variables, as well as the subsequent effect of those decisions on structural relationships, should occupy a central place in social scientific analyses. Game theoretical and related models can provide appropriate tools for such an endeavour.

None of this diminishes, however, the potential for the development of 'more scientific' approaches in political science. With the above-mentioned properties, mathematical equations based on sound theory can be validated by empirical tests, and can predict precise observations. They can provide not only knowledge and understanding of political phenomena, but also the best foundations for applied research in 'geological' fields such as electioneering, public administration, foreign affairs and others with a wide professional projection. 


\section{References}

Abramowitz, A. I. (2005) 'The Time-for-Change Model and the 2004 Presidential Election: A Post-Mortem and a Look Ahead', PS: Political Science \& Politics 38 (1): 31.

Arrow, K. (1985) 'Economic History: A Necessary Though Not Sufficient Condition for an Economist', American Economic Association Papers and Proceedings, pp. 320-3.

Campbell, J. E. (2004) 'Introduction', PS: Political Science \& Politics 37 (4): 733-5.

Crease, R. P. (2004) 'The Greatest Equations Ever', Physics World October: 19-23.

Farmelo, G. (ed.) (2002) It Must be Beautiful: Great Equations of Modern Science, New York: Granta Books.

Leigh, A., and Wolfers, J. (2006) 'Competing Approaches to Forecasting Elections: Economic Models, Opinion Polling and Prediction Markets', NBER Working Paper No. 12053.

Lewis-Beck, M. S., and Tien, C. (2004) 'Jobs and the Job of President: A Forecast for 2004', PS: Political Science \& Politics 37 (4): 753-8.

McGregor, J. P. (1993) 'Procrustus and the Regression Model: On the Misuse of the Regression Model', PS: Political Science \& Politics 26 (4): 801-4.

Malinvaud, E. (1970) 'The Consistency of Nonlinear Regressions', The Annals of Mathematical Statistics 41 (3): 956-69.

Prachowny, M. F. J. (1993) 'Okun's Law: Theoretical Foundations and Revised Estimates', The Review of Economics and Statistics 75 (2): 331-6.

Sargent, T. J. (1999) The Conquest of American Inflation, Princeton: Princeton University Press. 


\section{Note}

${ }^{1}$ I am very grateful to Rein Taagepera for pushing on this, as well as for fruitful exchanges with the other participants in the symposium at the ECPR Conference in Budapest, September 2005. I also acknowledge insightful hints made by José GarcíaMontalvo, Ramon Marimon and Andreu Mas-Colell. The usual caveats regarding the author's sole responsibility apply, this time perhaps even more than usual. 